\title{
Thorax and Abdomen Motion Analysis in Patients with Obstructive Diseases
}

Aïcha Laouani ${ }^{1 *}$, Sonia Rouatbi ${ }^{3}$, Saad Saguem ${ }^{1}$ and Pascale Calabrese ${ }^{2}$

${ }^{1}$ Laboratory of Biophysics, Faculty of Medicine of Sousse, Sousse, Tunisia

${ }^{2}$ Laboratory of Techniques de l'Ingénierie Médicale et de la Complexité - Informatique, Mathématiques et Applications, Grenoble (TIMC-IMAG) - PRETA team-CNRS:

UMR5525 - Université Joseph Fourier - Grenoble I, France

${ }^{3}$ Laboratory of Physiology, Faculty of Medicine of Sousse, Sousse, Tunisia

\begin{abstract}
Objective: We evaluated changes in bronchoconstriction by a new approach based on respiratory inductive plethysmography (RIP) signal analysis.

Methods: Thoracic and abdominal motions were recorded $(5 \mathrm{~min})$ by uncalibrated RIP in 44 adult subjects with a diagnosis of moderate bronchial obstruction (Obstructive group) and 50 healthy adult controls (Healthy group). In the Obstructive group, two series of measurements were performed before (Obstructive PRE) and after (Obstructive POST) a bronchodilation protocol. Airway resistance $\left(R_{a w}\right)$ and lung function data (forced vital capacity (FVC), forced expiratory volume in one second $\left(\mathrm{FEV}_{1}\right)$ and $\mathrm{FEV}_{1} / \mathrm{FVC}$ ) were measured with a body plethysmograph. A breath-bybreath analysis was performed to calculate distances between normalized thorax and abdomen RIP signals and a mean distance (D) was calculated for each recording.
\end{abstract}

Results: $D$ and $R_{a}$ were higher in the Obstructive group than in the Healthy group in both PRE and POST conditions. Both $D$ and $R_{a w}$ significantly decreased after bronchodilation in the Obstructive group. $D$ and $R_{a w}$ were also positively and significantly correlated in the Obstructive group in both PRE and POST conditions.

Conclusion: D, as calculated from signals recorded by RIP, appears to be a useful non-invasive parameter for continuous monitoring of changes in bronchoconstriction.

Keywords: Respiratory inductive plethysmography; Airway resistance; Bronchoconstriction; Thorax motions; Abdomen motions

\section{Introduction}

The most common method to detect the presence and severity of airflow limitation associated with obstructive lung disease is spirometry, considered as the gold standard pulmonary function testing. However, spirometry has some limitations: it is effort dependent and requires patient cooperation, it involves taking deep breaths, which can alter underlying airway resistance [1].

Although airway resistance $\left(\mathrm{R}_{\mathrm{aw}}\right)$ is seldom used to identify airway obstruction in clinical practice [2], its measurement becomes the only possibility of detecting airway obstruction in patients who cannot cooperate or perform reliable spirometry. Several methods, such as body plethysmography, oesophageal balloon, airflow perturbation techniques (including interrupter and oscillatory techniques), may be used to measure airway resistance [3]. Among these methods, body plethysmography is most widespread, and is believed to yield significant additional information compared to spirometry [4].

Respiratory Inductive Plethysmography (RIP) is another method that has the advantage over other techniques of being non-invasive. RIP allows recording of thorax and abdomen breathing movements using two sensors inserted in elastic bands surrounding thoracic and abdominal compartments. Analysis of these signals may be used to identify airway obstruction. The patient can thus be assessed during quiet breathing without a mouthpiece, and without the need of performing forced inspiratory and expiratory maximum motions. For infants with an acute upper airway obstruction, Sivan et al. [5] observed a high association between the degree of stridor and the thoracoabdominal asynchrony (TAA) quantified by phase angle analysis of the Lissajous figure from the output of an uncalibrated RIP. Allen et al. [6] reported a correlation between thoraco-abdominal phase angle and lung resistance measured with an oesophageal balloon in children. In the case of adults, Sackner et al. [7] did not observe any difference between healthy subjects and COPD patients when comparing TAA evaluated with phase angle. However, phase angle evaluation relies on the assumption that thorax and abdomen signals are sinusoidal, which is not always the case. Prisk et al. [8] compared different time domain methods of evaluating phase angle using simulated data when adding resistive loads during inspiration on anesthetized rhesus monkeys and concluded that cross-correlation and maximum linear correlation (methods that not depend on waveform shapes) are the most accurate and robust in measuring phase angles. In a recent mini-symposium, Seddon [9] stated that RIP "remains the most widely-used technique for semi-quantitative monitoring of chest wall movement and asynchrony".

In a preceding study [10], it was suggested that the addition of resistive loads entailed changes in the motion of abdominal and thoracic compartments. These changes were evaluated by calculating distances between thoracic and abdominal normalized RIP signals. It was found that these distances were correlated to the level of added resistive load in healthy subjects.

The present study was aimed to investigate a new approach for the evaluation of bronchoconstriction changes based on a breath-by-breath analysis of signals obtained by inductance plethysmography. For each breath, the distance between the thoracic and abdominal normalized RIP signals was calculated and averaged (D) over at least 30 breaths recorded on 1) healthy subjects and 2) patients with airway obstruction disease before and after bronchodilator administration. Comparison of $\mathrm{D}$ was carried out between healthy subjects and patients. In addition,

${ }^{*}$ Corresponding author: Aicha Laouani, Laboratory of Biophysics, Faculty of Medicine of Sousse, Sousse, Tunisia, Tel: +216 58850201; Fax: +216 73228994 E-mail: laouani_aicha@yahoo.fr

Received December 05, 2015; Accepted January 13, 2016; Published January 18, 2016

Citation: Laouani A, Rouatbi S, Saguem S, Calabrese P (2016) Thorax and Abdomen Motion Analysis in Patients with Obstructive Diseases. J Pulm Respir Med 6: 313. doi:10.4172/2161-105X.1000313

Copyright: @ 2016 Laouani A, et al. This is an open-access article distributed under the terms of the Creative Commons Attribution License, which permits unrestricted use, distribution, and reproduction in any medium, provided the original author and source are credited. 
for patients with airway obstruction disease, D was compared to $\mathrm{R}_{\mathrm{aw}}$ measured by body plethysmography both before and after bronchodilator administration.

\section{Materials and Methods}

\section{Subjects}

This cross-sectional analytic study was conducted in the Department of Physiology and Explorations in Farhat Hached Hospital of Sousse (Tunisia) in accordance with the Declaration of Helsinki. The local Ethics Committee of the Hospital approved the study protocol. All participants provided written consent and received a copy of their assessment results, which were also sent to their physicians.

The study was carried out on 44 (20 women) adult subjects with a diagnosis of moderate bronchial obstruction, as defined by comparison to reference values established by a local study [11]. This "Obstructive group" was compared to a "Healthy group" of 50 (29 women) healthy adult controls. Male and female subject data were not reported separately, as no additional information was gained by a separate study. Anthropometric data for the two groups are gathered in Table 1. Student's t-tests were carried out to compare the mean values of age, height, weight and body mass index (BMI) in the two groups. No significant difference was observed for any data.

All subjects were more than 18 years of age. The group with known airway obstruction contained subjects with a ratio of forced expiratory volume at the first second/forced vital capacity below the lower normal limit according to the American Thoracic Society guidelines [2]. The subjects with obstructive defects were clinically stable and did not show any signs of worsening symptoms or a need for increased medication or emergency care. The obstructive subjects had not required hospitalization within the previous 4 weeks. The medical treatments were recorded. The exclusion criteria were the following: age less than 18 years, cigarette smoking, alcohol abuse, renal failure, heart and coronary disease and current desensitization (therapeutic for reduction or elimination of an allergic reaction). Subjects having received $\beta$-agonists, oral or inhaled glucocorticoids, anti-histamines, anticholinergics, calcium, magnesium and beta-blockers during the previous 72 hours were also excluded. The healthy controls were volunteers non-smokers, over 20 years of age without a history of atopy and free from asthma, allergies, pulmonary tuberculosis or recent respiratory tract infection. The controls had normal pulmonary function tests and were free from any respiratory problems.

Imperfect performance of respiratory maneuvers was applied as an exclusion criterion in both study groups.

\section{Body plethysmography}

Pulmonary function measurements were performed with a body 285 plethysmograph (ZAN 500 Body II Mesgrerate GmbH, Germany) by carefully following international recommendations. The quiet breathing method was used in the present study to record total airway resistances. The following data were measured or calculated: airway resistance $\left(\mathrm{R}_{\mathrm{aw}}\right.$, in $\left.\mathrm{kPa} \cdot \mathrm{l}^{-1} \cdot \mathrm{sec}\right)$, forced vital capacity (FVC, l), forced expiratory volume in one second $\left(\mathrm{FEV}_{1}, \mathrm{l}\right)$ and the $\mathrm{FEV}_{1} / \mathrm{FVC}$ ratio.

\section{Respiratory inductive plethysmography (RIP)}

Thorax (THO) and abdomen (AB) breathing movements were recorded by RIP (Visuresp ${ }^{R}, \mathrm{RBI}$, France). The THO and AB signals were digitized at a sampling rate of $40 \mathrm{~Hz}$. Breaths were delimited using the algorithm developed by Bachy et al. [12] on a flow signal and applied to the RIP signal as the derivative of the filtered signal obtained from linear combination of both $\mathrm{THO}$ and $\mathrm{ABD}$ signals. Breaths involving swallowing, sigh, THO or AB signal drift were discarded from the analysis. A breath-by-breath analysis was then performed to calculate distances between $\mathrm{THO}$ and $\mathrm{AB}$ signals. Each $\mathrm{THO}$ and $\mathrm{AB}$ "cycle" included the same number $(m)$ of samples (same digitized sampling rate and duration). For each breath, the THO and AB signal amplitude was normalized to obtain a zero average and a standard deviation equal to one. The distance $\left(D_{\text {Breath }}\right)$ between normalized thorax $(n T H O)$ and abdominal $(n A B)$ signals was calculated over all

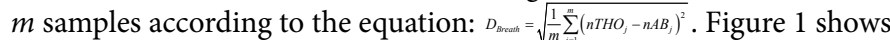
sensors of RIP incorporated in a wearable jacket $\left(\right.$ Visuresp $\left.^{\mathrm{R}}\right)$ at the thoracic and abdominal compartment level (A), thus recorded THO and ABD signals and delimitated breaths on the RIP signal (B) and calculation of $D_{\text {breath }}(\mathrm{C})$.

For each recording, the mean distance (D) was calculated over all selected (minimum 30) breaths.

\section{Experimental protocol}

All subjects (Healthy and Obstructive) underwent body plethysmography measurement followed by a five minute RIP recording at spontaneous breathing. Each patient then inhaled at $30 \mathrm{sec}$ intervals four successive doses of $100 \mathrm{mg}$ of short-acting $\beta_{2}$-agonist (Salbutamol) after a gentle and incomplete expiration and held the breath for 5-10 sec. Each patient again underwent body plethysmography measurement after 15 minutes, followed by a five minute RIP recording at spontaneous breathing. This described bronchodilation procedure is a standard protocol used in pulmonary function testing [2]. Thus, two conditions are to be taken into consideration in the Obstructive group: PRE (before bronchodilation) and POST (after bronchodilation).

\section{Data analysis}

All data have been expressed as the mean \pm SEM (Standard Error of the Mean). Student's t-test was used to compare the mean data between Healthy and Obstructive groups. Student's paired t-test was used to compare data within the Obstructive group before and after bronchodilation.

In the Obstructive group, Pearson correlation coefficient was used to evaluate the linear relationship between $\mathrm{D}$ and $\mathrm{R}_{\mathrm{aw}}$, as well as between $\mathrm{D}$ and spirometric data and between $\mathrm{R}_{\mathrm{aw}}$ and spirometric data in PRE and POST conditions. A binomial test was used to check the number of cases where bronchodilation entailed a decrease in $\mathrm{D}$ and $\mathrm{R}_{\mathrm{aw}}$

Significance was set at the 0.05 level.

\section{Results}

Figure 2 shows mean \pm SEM values of $D, R_{a w}$ and spirometric data $\left(\mathrm{FEV}_{1}, \mathrm{FVC}, \mathrm{FEV}_{1} / \mathrm{FVC}\right)$ for both Healthy and Obstructive groups in PRE and POST conditions. It can be seen that $\mathrm{D}$ and $\mathrm{R}_{\mathrm{aw}}$ values are lower in the Healthy group than in the Obstructive group in both PRE and POST conditions, whereas $\mathrm{FEV}_{1}, \mathrm{FVC}$, and $\mathrm{FEV}_{1} / \mathrm{FVC}$ are higher in the Healthy group than in the Obstructive group in both PRE and POST conditions. Comparing (Student's t-test) Healthy and Obstructive PRE on one hand, and Healthy and Obstructive POST on the other hand showed significant variations in all data $(\mathrm{p}<0.05)$, except FVC, which exhibited no significant difference between Healthy and Obstructive POST.

As expected in the Obstructive group, bronchodilation entailed a decrease in $\mathrm{D}$ and $\mathrm{R}_{\mathrm{aw}}$, while spirometric data increased. Comparison (Student's paired t-test) between Obstructive PRE and Obstructive POST showed significant difference in all data $(\mathrm{p}<0.05)$. 
Citation: Laouani A, Rouatbi S, Saguem S, Calabrese P (2016) Thorax and Abdomen Motion Analysis in Patients with Obstructive Diseases. J Pulm Respir Med 6: 313 doi:10.4172/2161-105X.1000313

A) Respiratory Inductive Plethysmography (RIP) jacke

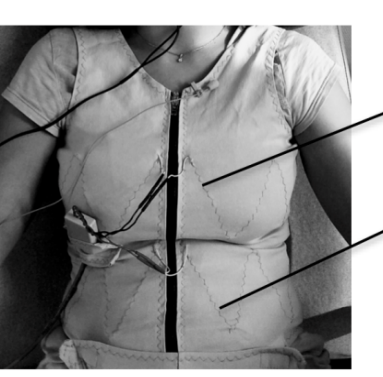

B) Thorax (THO) and abdomen (AB) RIP signals

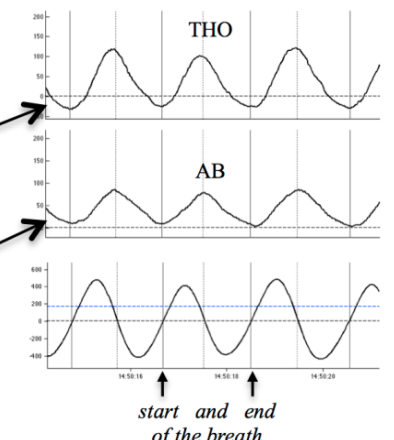

C) For each breath, including $m$ samples the distance $\left(D_{\text {Breath }}\right)$ between the normalized thorax (nTHO) and abdominal ( $\mathrm{AAB}$ ) signals was calculated:
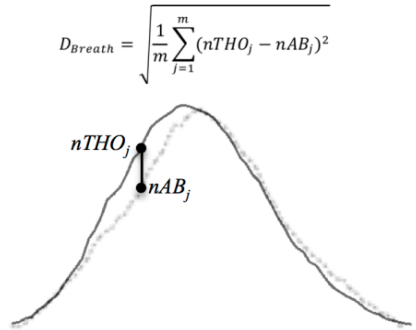

Figure 1: (A) Sensors of Respiratory Inductive Plethysmography (RIP) incorporated in a wearable jacket (Visuresp $R$ ) at the thoracic and abdominal compartment level. B) Breaths were delimited using the algorithm developed by Bachy et al. on a flow signal and applied to RIP signals: derivative of the filtered signal obtained from linear combination of THO and ABD signals. C) For one breath, ABD and THO normalized signals illustrating the calculation of the distance between thorax and abdomen motion $\left(\mathrm{D}_{\text {breath }}\right)$.

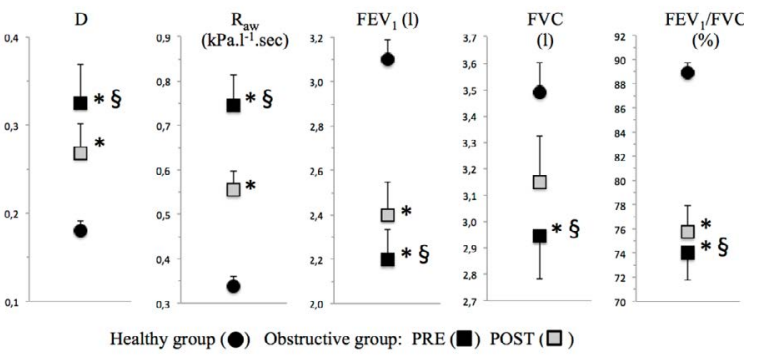

Figure 2: Mean \pm Standard Error of the Mean (SEM) values of distances (D) between the thorax and abdominal signals calculated over all selected (minimum 30$)$ breaths, airway resistance $\left(\mathrm{R}_{\text {aw }}\right.$ in $\left.\mathrm{kPa} . \mathrm{l}-1 . \mathrm{sec}\right)$ and spirometric data: forced vital capacity (FVC, in I), forced expiratory volume in one second (FEV, in I) and the $\mathrm{FEV}_{1} / \mathrm{FVC}$ ratio (in \%) for Healthy and Obstructive group before (PRE) and after (POST) bronchodilation. *'Significant differences (Student's t-test) between Healthy and Obstructive group (PRE and POST), §Significant differences (Student's t-test) between Obstructive PRE and POST.

Using a Binomial test, it was found that it was a significant number of subjects showing a decrease in $\mathrm{D}(28$ subjects over $44, \mathrm{p}=0.024)$ and $\mathrm{R}_{\mathrm{aw}}$ (32 subjects over $44, \mathrm{p}=0.001$ ) values after bronchodilation. It must be underlined at this point that these subjects did not systematically exhibit a simultaneous decrease in both $\mathrm{D}$ and $\mathrm{R}_{\mathrm{aw}}$ values.

We did however calculate correlation coefficients between $\mathrm{D}$ and $\mathrm{R}_{\mathrm{aw}}$, as well as between $\mathrm{D}$ and spirometric data and between $\mathrm{R}_{\mathrm{aw}}$ and spirometric data, in PRE (Table 2) and POST (Table 3) conditions within the Obstructive group. A positive correlation exists between $\mathrm{D}$ and $\mathrm{R}_{\text {aww }}$ both in PRE and POST conditions, but the correlations between $\mathrm{D}$ and spirometric data were negative and significant for all variables, except for FVC in Obstructive PRE condition. $\mathrm{R}_{\mathrm{aw}}$ was significantly and negatively correlated with all spirometric data in both conditions.

\section{Discussion}

The main result of this study is that the distance D calculated between thorax and abdomen normalized signals, as recorded by respiratory inductive plethysmography may provide information on bronchoconstriction. Indeed, 1) D was significantly higher in the Obstructive group in both PRE and POST conditions than in the Healthy group and 2) in the Obstructive group, $\mathrm{D}$ and $\mathrm{R}_{\mathrm{aw}}$ were

\begin{tabular}{|c|c|c|c|}
\hline & Healthy $\mathbf{n = 5 0}$ & Obstructive $\mathbf{n = 4 4}$ & $\mathbf{p}$ \\
\hline Age (years) & $44.3 \pm 1.8$ & $47.0 \pm 2.1$ & 0.34 \\
\hline Height $(\mathrm{m})$ & $1.66 \pm 0.01$ & $1.64 \pm 0.02$ & 0.53 \\
\hline Weight $(\mathrm{kg})$ & $76.2 \pm 1.7$ & $77.5 \pm 3.0$ & 0.69 \\
\hline BMI $\left(\mathrm{kg} \cdot \mathrm{m}^{-2}\right)$ & $27.8 \pm 0.6$ & $28.9 \pm 1.2$ & 0.39 \\
\hline
\end{tabular}

BMI: Body Mass Index; $p$ : p-value corresponding to comparison between the two groups (Student's t-test).

Table 1: Anthropometric characteristics (mean \pm SEM) of the Healthy (control) and Obstructive (patients with a diagnosis of moderate bronchial obstruction) group.

\begin{tabular}{|c|c|c|c|c|}
\hline & $\mathbf{R}_{\mathrm{aw}}$ & FEV $_{1}$ & FVC & $\mathrm{FEV}_{1} / \mathrm{FVC}$ \\
\hline$D$ & $0.48^{*}$ & $-0.37^{*}$ & -0.27 & $-0.34^{*}$ \\
\hline $\mathrm{R}_{\mathrm{aw}}$ & & $-0.55^{*}$ & $-0.38^{*}$ & $-0.57^{\star}$ \\
\hline
\end{tabular}

Table 2: Correlation coefficient values between thoraco-abdominal distance (D) and airway resistance $\left(R_{2}\right)$, between $D$ and spirometric data and between $R_{a w}$ and spirometric data, in Obstructive group before bronchodilation (Obstructive PRE).

\begin{tabular}{|c|c|c|c|c|}
\hline & $\mathbf{R}_{\mathrm{aw}}$ & FEV $_{1}$ & FVC & FEV $_{\mathbf{1}} /$ FVC \\
\hline $\mathrm{D}$ & $0.39^{*}$ & $-0.44^{*}$ & $-0.38^{*}$ & $-0.35^{*}$ \\
\hline $\mathrm{R}_{\mathrm{aw}}$ & & $-0.49^{*}$ & $-0.37^{*}$ & $-0.43^{*}$ \\
\hline
\end{tabular}

'Significant correlation (Pearson) between the two considered data $(p<0.05)$;

$\mathrm{FEV}_{1}$ : Forced expiratory volume in one second, FVC: forced vital capacity.

Table 3: Correlation coefficient values between thoraco-abdominal distance (D) and airway resistance $\left(R_{a w}\right)$, between $D$ and spirometric data and between $R_{a w}$ and spirometric data, in Obstructive group after bronchodilation (Obstructive POST).

correlated in both conditions.

Healthy and Obstructive groups showed no significant difference in anthropometric data (age, height, weight and BMI). The spirometric data, $\mathrm{R}_{\mathrm{aw}}$ and $\mathrm{D}$ were significantly different between Healthy and Obstructive groups in PRE condition, and there still was a significant difference between Healthy and Obstructive groups in POST condition, except for FVC. 
Although spirometry is considered as the gold standard to detect airflow limitation in obstructive diseases, recent articles revisited the contribution of other data, such as airway resistance measured by various methods [1] and thorax and abdomen motions measured by respiratory inductive plethysmography [9]. Interest for separate thoracic and abdominal motions during breathing was introduced in the 1960s. Indeed, Agostoni and Mognoni initiated the measure of chest wall deformation [13], and Konno and Mead evaluated the separate volume of the two compartments [14]. Since then, a large variety of methods have been employed for measuring thorax and abdomen motions, as reviewed by Seddon [9]. Since the 1990s, RIP has been the most common method in both adults and children, particularly for evaluating thoraco-abdominal asynchrony [5,6,15-23]. Thoracic and abdominal motions have been analysed to quantitatively evaluate thoraco-abdominal asynchrony (TAA) defined by Prisk et al. [8] as "the non-coincident motion of rib cage and abdomen during breathing". Several methods have been used to quantify TAA with or without calibrating RIP. On one hand, the values calculated using uncalibrated RIP can be either phase angle (Lissajous figures: X-Y plots of thorax versus abdomen) [5,16-18,23], or percent time paradoxical to tidal volume (during inspiration, expiration or total breath) [24] and phase relation during total breath (percentage of total breath duration where thorax and abdomen are asynchronously moving) [20,22]. On the other hand, the values calculated with calibrated RIP can be either asynchrony index [25], laboured breathing index (maximal compartmental amplitude-sum of maximal excursion of thorax and abdomen- as proportion of tidal volume) [7] or rib cage contribution to tidal volume (maximum excursion of thorax as a percentage of tidal volume) [26].

In our study, distances between the thoracic and abdominal normalized signals serve to evaluate differences in these two compartments motion. This was calculated breath-by-breath on five minutes recordings. Thus, the mean value (D) may be considered as calculated over a "steady-state" and we assumed that it provides a satisfactory evaluation of motion differences between thoracic and abdominal compartments induced by bronchoconstriction. However, concerning $\mathrm{R}_{\mathrm{aw}}$ data they result from a single measurement. This may account for the observed discrepancies between $\mathrm{D}$ and $\mathrm{R}_{\mathrm{aw}}$, such as the fact that following a bronchodilation, more people showed a decrease in $\mathrm{R}_{\mathrm{aw}}$ rather than a decrease in $\mathrm{D}$. Indeed, similar to $\mathrm{R}_{\mathrm{aw}}$, $\mathrm{D}$ was higher in the Obstructive group as compared to the Healthy group, both before and after bronchodilation. The fact that in the Obstructive group (both conditions), $\mathrm{D}$ and $\mathrm{R}$ were significantly correlated and that they also correlated with spirometric data indicates that $\mathrm{D}$ as well as $\mathrm{R}_{\mathrm{aw}}$ may assess bronchoconstriction.

These results suggest that beside classical methods [1,2] used to evaluate bronchoconstriction, inductive plethysmography provides relevant information on bronchoconstriction with several notable advantages. Indeed, RIP is a non-invasive method to record thoracic and abdominal motion without mouthpiece. Furthermore, the analysis is performed over signals acquired during quiet breathing. Thus, since no subject cooperation or specific handling is required, measurements can be easily repeated. In addition, RIP may be calibrated to provide volume and flow data [27-29]. In the device (Visuresp ${ }^{\circ}$ ) used in this study, sensors have been incorporated in a wearable jacket and maintained in a fixed position (Figure 1A) allowing data comparison on continuous recordings. Prior calibration in various postures [30] may then be applied in longitudinal measurements of respiratory function by RIP [22]. RIP monitoring can thus be envisaged to assess changes in bronchoconstriction induced by therapeutics, environmental variations or various conditions such as sleep.

\section{Conclusion}

The breath-by-breath distance between thorax and abdomen normalized signals recorded by respiratory inductive plethysmography and averaged over a 5 minute period may represent a new method of RIP use for bronchoconstriction changes evaluation.

\section{Conflict of Interests}

The authors declare that there is no conflict of interests regarding the publication of this paper.

\section{References}

1. Kaminsky DA (2012) What does airway resistance tell us about lung function? Respir Care 57: 85-96.

2. Pellegrino R, Viegi G, Brusasco V, Crapo RO, Burgos F, et al. (2005) Interpretative strategies for lung function tests. Eur Respir J 26: 948-968.

3. Blonshine S, Goldman MD (2008) Optimizing performance of respiratory airflow resistance measurements. Chest 134: 1304-1309.

4. Criée CP, Sorichter S, Smith HJ, Kardos P, Merget R, et al. (2011) Body plethysmography--its principles and clinical use. Respir Med 105: 959-971.

5. Sivan Y, Deakers TW, Newth CJ (1990) Thoracoabdominal asynchrony in acute upper airway obstruction in small children. Am Rev Respir Dis 142: 540-544.

6. Allen JL, Greenspan JS, Deoras KS, Keklikian E, Wolfson MR, et al. (1991) Interaction between chest wall motion and lung mechanics in normal infants and infants with bronchopulmonary dysplasia. Pediatr Pulmonol 11: 37-43.

7. Sackner MA, Gonzalez H, Rodriguez M, Belsito A, Sackner DR, et al. (1984) Assessment of asynchronous and paradoxic motion between rib cage and abdomen in normal subjects and in patients with chronic obstructive pulmonary disease. Am Rev Respir Dis 130: 588-593.

8. Prisk GK, Hammer J, Newth CJ (2002) Techniques for measurement of thoracoabdominal asynchrony. Pediatr Pulmonol 34: 462-472.

9. Seddon $P$ (2015) Options for assessing and measuring chest wall motion Paediatr Respir Rev 16: 3-10.

10. Calabrese $P$ (1998) Study for a non-invasive method of respiratory resistance measurement- Effects of resistive loading on breathing pattern and heart rate variability, Université Joseph Fourier (Grenoble, France.

11. Tabka Z, Hassayoune H, Guenard H, Zebidi A, Commenges D, et al. (1995) [Spirometric reference values in a Tunisian population]. Tunis Med 73: 125-131.

12. Bachy JP, Eberhard A, Baconnier P, Benchetrit G (1986) A program for cycleby-cycle shape analysis of biological rhythms. Application to respiratory rhythm. Comput Methods Programs Biomed 23: 297-307.

13. Agostoni E, Mognoni P (1966) Deformation of the chest wall during breathing efforts. J Appl Physiol 21: 1827-1832.

14. Konno K, Mead J (1967) Measurement of the separate volume changes of rib cage and abdomen during breathing. J Appl Physiol 22: 407-422.

15. Allen JL, Wolfson MR, McDowell K, Shaffer TH (1990) Thoracoabdominal asynchrony in infants with airflow obstruction. Am Rev Respir Dis 141: 337-342.

16. Davis GM, Cooper DM, Mitchell I (1993) The measurement of thoracoabdominal asynchrony in infants with severe laryngotracheobronchitis. Chest 103: $1842-1848$

17. Hammer J, Newth CJ (2009) Assessment of thoraco-abdominal asynchrony Paediatr Respir Rev 10: 75-80.

18. Hammer J, Newth CJ, Deakers TW (1995) Validation of the phase angle technique as an objective measure of upper airway obstruction. Pediatr Pulmonol 19: 167-173.

19. Selbie RD, Fletcher M, Arestis N, White R, Duncan A, et al. (1997) Respiratory function parameters in infants using inductive plethysmography. Med Eng Phys 19: $501-511$.

20. Upton J, Brodie D, Beales D, Richardson J, Jack S, et al. (2012) Correlation between perceived asthma control and thoraco-abdominal asynchrony in primary care patients diagnosed with asthma. J Asthma 49: 822-829. 
Citation: Laouani A, Rouatbi S, Saguem S, Calabrese P (2016) Thorax and Abdomen Motion Analysis in Patients with Obstructive Diseases. J Pulm Respir Med 6: 313. doi:10.4172/2161-105X.1000313

21. Chien JY, Ruan SY, Huang YC, Yu CJ, Yang PC (2013) Asynchronous thoracoabdominal motion contributes to decreased 6-minute walk test in patients with COPD. Respir Care 58: 320-326.

22. Mayer OH, Clayton RG Sr, Jawad AF, McDonough JM, Allen JL (2003) Respiratory inductance plethysmography in healthy 3- to 5-year-old children. Chest 124: 1812-1819.

23. Reber A, Bobbià SA, Hammer J, Frei FJ (2001) Effect of airway opening manoeuvres on thoraco-abdominal asynchrony in anaesthetized children. Eur Respir J 17: 1239-1243.

24. Benameur M, Goldman MD, Ecoffey C, Gaultier C (1993) Ventilation and thoracoabdominal asynchrony during halothane anesthesia in infants. J Appl Physiol (1985) 74: 1591-1596.

25. Warren RH, Alderson SH (1994) Chest wall motion in neonates utilizing respiratory inductive plethysmography. J Perinatol 14: 101-105.
26. Hershenson MB, Colin AA, Wohl ME, Stark AR (1990) Changes in the contribution of the rib cage to tidal breathing during infancy. Am Rev Respir Dis 141: 922-925.

27. Gonzalez H, Haller B, Watson HL, Sackner MA (1984) Accuracy of respiratory inductive plethysmograph over wide range of rib cage and abdominal compartmental contributions to tidal volume in normal subjects and in patients with chronic obstructive pulmonary disease. Am Rev Respir Dis 130: 171-174.

28. Eberhard A, Calabrese P, Baconnier P, Benchetrit G (2001) Comparison between the respiratory inductance plethysmography signal derivative and the airflow signal. Adv Exp Med Biol 499: 489-494.

29. Calabrese P, Besleaga T, Eberhard A, Vovc V, Baconnier P (2007) Respiratory inductance plethysmography is suitable for voluntary hyperventilation test. Conf Proc IEEE Eng Med Biol Soc 2007: 1055-1057.

30. Sackner MA, Watson H, Belsito AS, Feinerman D, Suarez M, et al. (1989) Calibration of respiratory inductive plethysmograph during natural breathing. $J$ Appl Physiol 66: 410-420. 